\title{
Genetic Analysis in Pearl millet Using Line x Tester Design
}

\author{
K. N. Patel ${ }^{\text {* }}$, K. K. Dhedhi ${ }^{2}$, H. Ramani ${ }^{2}$ and U. U. Vaghela ${ }^{3}$ \\ ${ }^{1}$ Department of Genetics and Plant Breeding, College of Agriculture, JAU, Junagadh, India \\ ${ }^{2}$ Main Pearl Millet Research Station, JAU, Junagadh, India \\ ${ }^{3}$ Department of Genetics and Plant Breeding, N. M. College of Agriculture, \\ NAU, Navsari, India \\ *Corresponding author
}

\section{A B S T R A C T}

\section{Keywords}

Pearlmillet, L x T

design, General combining ability, Specific combining ability

Article Info

Accepted:

12 December 2020

Available Online:

10 January 2021
Four cytoplasmic male sterile lines were crossed with nine restorers to develop 36 hybrids using line $\mathrm{x}$ tester design to study combining ability in pearl millet for twelve different characters and evaluated at main pearlmillet research station, JAU, Jamnaghar in kharif 2018. Analysis of variance showed highly significant differences among the genotypes for all the traits. Thus, the results quantified the considerable amount of genetic diversity exist among genotypes for the different traits under study. Estimates of $\sigma^{2}$ sca were greater than the corresponding $\sigma^{2}$ gca for ear head girth, ear head weight, 1000-grain weight, dry fodder yield per plant, grain yield per plant and harvest index, indicated the preponderance of non-additive gene effects. While for rest of the characters, the ratio of $\sigma^{2} \mathrm{gca} / \sigma^{2} \mathrm{sca}$ was found greater than unity, suggested the preponderance of additive gene effects for the express of these traits. Females $\mathrm{JMSA}_{4}-20172$ and $\mathrm{ICMA}_{1}-15666$ and males J-2598, J2605, 92-SB-18 and 72-SB-18 were found good general combiners for grain yield per plant and also some desirable traits. $\mathrm{JMSA}_{5}-20175$ x J-2598 and $\mathrm{JMSA}_{4}-20172$ x J-2591 manifested significant and high desirable sca effects for grain yield per plant (Table 5) along with significant positive sca effect for atleast one character, with involving at least one poor and one good general combiners.

\section{Introduction}

Pearl millet is a cereal crop that thrives in the arid and semi-arid tropical regions of Asia and Africa. Pearl millet grain is highly nutritive, staple food and the primary source of calories for millions of people who are inhabitants of world's driest and hottest regions of poor farmer communities. It is a highly cross-pollinated crop with protogynous flowering and has wind borne pollination mechanism, which fulfill one of the essential biological requirements for hybrid development. Pearl millet is commonly known as bajra, cat tail millet, spiked millet and bulrush millet in different parts of the world, which is believed to be originated in Africa. The amino acid profile of pearl millet is more balanced than wheat and rice and many other cereals, and low glycemic index 
(Singh et al., 1999 and Sehgal et al., 2004). In heterosis breeding identification and assessment of the parental combinations with respect to their general and specific combining abilities and gene actions involved in the inheritance of grain yield and various component characters are of upmost importance for a successful hybridization programme. Recognition of a potential hybrid combination through the magnitude and direction of heterotic behavior is of paramount importance. So, information on the magnitude and nature of prevalent genetic variation is essentially needed to infer about genetic potential of a particular population.

\section{Materials and Methods}

The experimental material comprising by four cytoplasmic male sterile lines (JMSA ${ }_{4}-20172$, $\mathrm{JMSA}_{5}-20175, \quad \mathrm{JMSA}_{1}-20181, \quad \mathrm{ICMA}_{1}$ 15666), nine restorers (J-2584, J-2591, J2598, J-2605, 52-SB-18, 72-SB-18, 92-SB$18,120-\mathrm{SB}-18$, their resulting 36 crosses and one standard check GHB-732 were evaluated in Randomized Block Design with three replications during kharif 2018. Observations were recorded on 12 characters and their mean values were subjected to analysis of variance and combining ability. The crosses were made during summer 2018 at Main Pearl Millet Research Station, Junagadh Agricultural University, Jamnagar using line $\mathrm{x}$ tester mating design.

\section{Results and Discussion}

\section{Combining ability analysis}

The analysis of variance showed highly significant differences among the genotypes for all the traits. Thus, the results quantified the considerable amount of genetic diversity exist among genotypes for the different traits under study due to presence of highly significant difference among genotypes. The genotypic variance was further partitioned into different source of variances.

Mean squares due to females were significant for all the studied characters except harvest index among all the characters while, the male parents differed significantly for all the twelve characters under studied. This concluded the presence of genetic diversity in both the females and males parents included in the investigation. The males and females were completely differed from each other for days to $50 \%$ flowering, number of nodes on main stem, ear head girth, plant height, days to maturity and dry fodder yield per plant as evident from significance of mean squares due to females $v s$ males. The differences among parents and crosses and parents vs crosses were also found highly significant for all characters under investigation(Table 1).Similar observations were also made by Patel (2012), Mungra (2014), Acharya (2017),Katba (2017) and Badurkar et al., (2018).

Indication of table 2 shows analysis of variance for combining ability for all twelve studied characters, In which mean squares due to females were significant for days to $50 \%$ flowering, number of nodes on main stem, ear head length, ear head weight, plant height, days to maturity, dry fodder yield per plant and grain yield per plant. While, the mean squares due to males were significant for days to $50 \%$ flowering, ear head length, plant height and days to maturity. This indicated significance contribution of both parents towards total variation due to general combining ability (gca). The mean squares due to females $x$ males were found significant for all the characters studied except ear head length. The estimates of $\sigma^{2}$ sca were greater than the corresponding $\sigma^{2}$ gca for ear head girth, ear head weight, 1000-grain weight, dry fodder yield per plant, grain yield per plant and harvest index, these resulted in less than 
unity ratio of $\sigma^{2} \mathrm{gca} / \sigma^{2} \mathrm{sca}$, indicated the preponderance of non-additive gene effects. Whereas, for the rest of the characters, the ratio of $\sigma^{2} \mathrm{gca} / \sigma^{2}$ sca was found greater than unity, suggested the preponderance of additive gene effects for the express of these traits. These findings were in accordance with previous studies of Nandaniya (2016), Krishnan et al., (2017), Solanki et al., (2017), Badurkar et al., (2018), Ladumor et al., (2018) and Kumawat et al., (2019).

\section{GCA and SCA effects}

The best performing parents (females and males) and cross combinations on the basis of GCA and SCA effects revealed that none of the parents was found good general combiner for all the characters which suggested breeding for these characters would be effective when material is tested over a wide range of environments. Female parent $\mathrm{JMSA}_{4}-20172$ noticed good general combiner for grain yield per plant including good gca for number of nodes on main stem, ear head length, ear head weight, plant height and dry fodder yield per pant. Another, female parent ICMA $_{1}-15666$ was appeared to be good source of gene for grain yield per plant as well as for number of effective tillers per plant, ear head girth, ear head weight, 1000grain weight and dry fodder yield per plant. Moreover, if we discuss about general combining of male parents, J-2598 found good combiner for grain yield was also recorded good gca effects in favorable direction for number of nodes on main stem, ear head length, ear head weight, plant height and dry fodder yield per plant. J-2605 found good combiner for plant height only other than grain yield. Likewise, parent 72-SB-18 was noted to have greater potential to transmit useful genes for grain yield per plant, days to $50 \%$ flowering, ear head length and days to maturity. Male parent 92-SB-18 manifested good general combiner for grain yield per plant, number of nodes on main stem, ear head girth, plant height, days to maturity and dry fodder yield per plant (Table 3 ). Thus, the parents were good general combiner for grain yield per plant also showed good general combining ability for one or more component traits. These findings are in concurrence with results of Jethava et al., (2012), Mungra (2014), Acharya (2017), Badurkar et al., (2018), Gavali et al., (2018) and Kumawat et al., (2019) in pearl millet. In the present investigation, among the parents, J-2598 manifested the maximum positive gca effect for the grain yield per plant followed by $\mathrm{JMSA}_{4}-20172$ and J-2605 which can be used in further breeding programme to improve yield and it's component characters.

The estimates of sca effect revealed that none of the crosses was consistently superior for all the traits. Out of 36 crosses studied, a number of crosses showed significant sca effects in desired direction for grain yield per plant (6), days to $50 \%$ flowering (8), number of nodes on main stem (2), number of effective tillers per plant (4), ear head girth (7), ear head weight (3), plant height (4), days to maturity (5), 1000-grain weight (3), dry fodder yield per plant (1) and harvest index (1) (Table 4).

Out of five top most high yielding cross combinations, only two cross combinations viz., JMSA $\mathrm{J}_{5}-20175$ x J-2598 and $\mathrm{JMSA}_{4}{ }^{-}$ 20172 x J-2591 manifested significant and high desirable sca effects for grain yield per plant (Table 5) along with significant positive sca effect for atleast one character, with involving at least one poor and one good general combiners. Other crosses like ICMA $_{1-}$ 15666 x 92-SB-18, ICMA 1 -15666 x J-2605 and $\mathrm{JMSA}_{4}-20172 \times 52-\mathrm{SB}-18$ having high mean value for grain yield and having significant positive heterobeltiosis and standard heterosis involved good $\mathrm{x}$ good, good x good and good x average combiners, respectively. 
Table.1 Analysis of variance for experimental design for different characters in pearlmillet

\begin{tabular}{|c|c|c|c|c|c|c|c|c|c|c|c|c|c|}
\hline Source & d.f. & $\begin{array}{c}\text { Days to } \\
50 \% \\
\text { flowering }\end{array}$ & $\begin{array}{c}\text { Number } \\
\text { of nodes } \\
\text { on main } \\
\text { stem }\end{array}$ & $\begin{array}{c}\text { Number of } \\
\text { effective } \\
\text { tillers/plant }\end{array}$ & $\begin{array}{c}\text { Ear } \\
\text { head } \\
\text { length } \\
(\mathrm{cm})\end{array}$ & $\begin{array}{l}\text { Ear } \\
\text { head } \\
\text { girth } \\
(\mathrm{cm})\end{array}$ & $\begin{array}{c}\text { Ear head } \\
\text { weight } \\
\text { (g) }\end{array}$ & $\begin{array}{c}\text { Plant } \\
\text { height } \\
\text { (cm) }\end{array}$ & $\begin{array}{l}\text { Day to } \\
\text { maturity }\end{array}$ & $\begin{array}{c}\text { 1000- } \\
\text { grain } \\
\text { weight }(\mathrm{g})\end{array}$ & $\begin{array}{c}\text { Dry } \\
\text { fodder } \\
\text { yield/plant } \\
(\mathrm{g})\end{array}$ & $\begin{array}{c}\text { Grain } \\
\text { yield/ } \\
\text { plant (g) }\end{array}$ & $\begin{array}{c}\text { Harvest } \\
\text { index }(\%)\end{array}$ \\
\hline Replications & 2 & 0.06 & 0.04 & 0.03 & $10.46^{*}$ & 0.15 & 15.43 & 97.33 & $5.19 *$ & $3.16^{*}$ & 83.21 & 59.57 & 26.03 \\
\hline Genotypes & 48 & $20.44 * *$ & $4.57 * *$ & $0.45^{* *}$ & $27.57 * *$ & $1.40^{* *}$ & $1765.28 * *$ & $3307.08 * *$ & $36.50 * *$ & $8.39 * *$ & $1195.71^{* *}$ & $1030.04 * *$ & $84.56^{* *}$ \\
\hline Parents (P) & 12 & $34.42 * *$ & $3.93 * *$ & $0.32 * *$ & $41.48 * *$ & $1.66 * *$ & $367.44 * *$ & $4640.19 * *$ & $40.48 * *$ & $7.48 * *$ & $1083.22 * *$ & $361.98 * *$ & $65.76^{* *}$ \\
\hline Females & 3 & $13.19 * *$ & $5.23 * *$ & $0.43 * *$ & $48.73 * *$ & $0.64 * *$ & $188.33^{*}$ & $7462.37 * *$ & $10.53 * *$ & $4.49^{* *}$ & $957.50 * *$ & $75.68 *$ & 2.58 \\
\hline Males & 8 & $38.83 * *$ & $1.12 * *$ & $0.18^{*}$ & $43.85 * *$ & $1.63 * *$ & $478.77 * *$ & $2089.70 * *$ & $43.98 * *$ & $9.27 * *$ & $1203.35^{* *}$ & $510.11 * *$ & $97.33 * *$ \\
\hline $\begin{array}{c}\text { Female vs } \\
\text { Male }\end{array}$ & 1 & $62.83 * *$ & $22.47 * *$ & 1.07 & 0.80 & $5.02 * *$ & 14.04 & $16577.52 * *$ & $102.31 * *$ & 2.01 & $499.22 * *$ & 35.79 & 2.74 \\
\hline Crosses (C) & 35 & $15.82 * *$ & $3.74 * *$ & $0.45 * *$ & $13.24 * *$ & $1.01 * *$ & $686.96^{* *}$ & $1522.01 * *$ & $34.39 * *$ & $5.81 * *$ & $688.47 * *$ & $515.49 * *$ & $50.70 * *$ \\
\hline P. Vs C. & 1 & $14.33 * *$ & $41.48 * *$ & $2.31 * *$ & $361.84 * *$ & $11.74 * *$ & $56280.72 * *$ & $49787.59 * *$ & $62.58 * *$ & $109.65^{* *}$ & $20299.18 * *$ & $27056.04^{* *}$ & $1495.50 * *$ \\
\hline Error & 96 & 0.56 & 0.30 & 0.07 & 2.69 & 0.09 & 63.65 & 78.19 & 1.31 & 1.01 & 91.57 & 27.24 & 15.34 \\
\hline
\end{tabular}

*, ** Significant at $5 \%$ and $1 \%$ levels, respectively, when tested against error mean square

Table.2 Analysis of variance for combining ability and various components for different characters in pearlmillet

\begin{tabular}{|c|c|c|c|c|c|c|c|c|c|c|c|c|c|}
\hline Source & d.f. & $\begin{array}{c}\text { Days to } \\
50 \% \\
\text { flowering }\end{array}$ & $\begin{array}{c}\text { Number } \\
\text { of nodes } \\
\text { on main } \\
\text { stem }\end{array}$ & $\begin{array}{c}\text { Number of } \\
\text { effective } \\
\text { tillers/plant }\end{array}$ & $\begin{array}{c}\text { Ear } \\
\text { head } \\
\text { length } \\
(\mathrm{cm})\end{array}$ & $\begin{array}{l}\text { Ear } \\
\text { head } \\
\text { girth } \\
(\mathrm{cm})\end{array}$ & $\begin{array}{c}\text { Ear head } \\
\text { weight } \\
\text { (g) }\end{array}$ & $\begin{array}{c}\text { Plant } \\
\text { height } \\
\text { (cm) }\end{array}$ & $\begin{array}{c}\text { Day to } \\
\text { maturity }\end{array}$ & $\begin{array}{c}\text { 1000- } \\
\text { grain } \\
\text { weight }(\mathrm{g})\end{array}$ & $\begin{array}{c}\text { Dry } \\
\text { fodder } \\
\text { yield/plant } \\
\text { (g) }\end{array}$ & $\begin{array}{c}\text { Grain } \\
\text { yield/ } \\
\text { plant (g) }\end{array}$ & $\begin{array}{c}\text { Harvest } \\
\text { index }(\%)\end{array}$ \\
\hline Replications & 2 & 0.36 & 0.07 & 0.01 & 8.22 & 0.09 & 6.40 & 62.08 & $5.62 *$ & $4.62 *$ & 150.22 & 55.86 & 42.00 \\
\hline Females & 3 & $24.13 * *$ & $20.95 * *$ & 0.81 & $58.53 * *$ & 1.82 & $2064.02 *$ & $7339.34 * *$ & $97.71 * *$ & 11.91 & $2797.85^{* *}$ & $1324.75^{*}$ & 22.70 \\
\hline Males & 8 & $45.81 * *$ & 3.58 & 0.12 & $24.55 * *$ & 0.54 & 713.39 & $2148.65 * *$ & $73.74 * *$ & 3.16 & 621.61 & 721.44 & 93.27 \\
\hline $\begin{array}{c}\text { Females x } \\
\text { Males }\end{array}$ & 24 & $4.78 * *$ & $1.63 * *$ & $0.50 * *$ & 3.81 & $1.07 * *$ & $506.02 * *$ & $585.95^{* *}$ & $13.36 * *$ & $5.92 * *$ & $447.07 * *$ & $345.68 * *$ & $40.00 * *$ \\
\hline Error & 70 & 0.49 & 0.32 & 0.07 & 2.76 & 0.07 & 83.12 & 82.08 & 1.31 & 0.97 & 100.12 & 31.11 & 14.88 \\
\hline$\sigma^{2} f$ & & 0.88 & 0.76 & 0.03 & 2.07 & 0.0645 & 73.37 & 268.79 & 3.57 & 0.41 & 99.92 & 47.91 & 0.29 \\
\hline$\sigma^{2} m$ & & 3.78 & 0.27 & 0.01 & 1.82 & 0.04 & 52.52 & 172.21 & 6.03 & 0.18 & 43.46 & 57.53 & 6.53 \\
\hline$\sigma^{2}$ gca & & 1.77 & 0.61 & 0.20 & 1.97 & 0.06 & 66.96 & 239.07 & 4.33 & 0.34 & 82.54 & 50.87 & 2.21 \\
\hline$\sigma^{2}$ sca & & 1.43 & 0.44 & 0.15 & 0.35 & 0.33 & 140.96 & 167.96 & 4.01 & 1.65 & 115.65 & 104.85 & 8.37 \\
\hline$\sigma^{2}$ gca $/ \sigma^{2}$ sca & & 1.24 & 3.20 & 1.39 & 5.66 & 0.17 & 0.47 & 1.42 & 1.08 & 0.20 & 0.71 & 0.49 & 0.26 \\
\hline
\end{tabular}

$*$,** Significant at $5 \%$ and $1 \%$ levels, respectively, when tested against error mean square 
Table.3 Estimates of general combining ability effects of parents for different characters in pearl millet

\begin{tabular}{|c|c|c|c|c|c|c|c|c|c|c|c|c|c|}
\hline $\begin{array}{l}\text { Sr. } \\
\text { No. }\end{array}$ & Parents & $\begin{array}{c}\text { Days to } 50 \% \\
\text { flowering }\end{array}$ & $\begin{array}{c}\text { No. of } \\
\text { nodes on } \\
\text { main stem }\end{array}$ & $\begin{array}{c}\text { No. of } \\
\text { effective } \\
\text { tillers/plant }\end{array}$ & $\begin{array}{c}\text { Ear } \\
\text { head } \\
\text { length } \\
(\mathrm{cm})\end{array}$ & $\begin{array}{c}\text { Ear head } \\
\text { girth } \\
(\mathrm{cm})\end{array}$ & $\begin{array}{c}\text { Ear head } \\
\text { weight (g) }\end{array}$ & $\begin{array}{c}\text { Plant height } \\
(\mathrm{cm})\end{array}$ & $\begin{array}{l}\text { Days to } \\
\text { maturity }\end{array}$ & $\begin{array}{c}\text { 1000-grain } \\
\text { weight (g) }\end{array}$ & $\begin{array}{c}\text { Dry } \\
\text { fodder } \\
\text { yield/pla } \\
\text { nt }(g)\end{array}$ & $\begin{array}{c}\text { Grain } \\
\text { yield/ } \\
\text { plant (g) }\end{array}$ & $\begin{array}{c}\text { Harvest } \\
\text { index } \\
(\%)\end{array}$ \\
\hline \multicolumn{14}{|c|}{ Females } \\
\hline 1 & $\mathrm{JMSA}_{4} 20172$ & $0.57 * *$ & $1.13 * *$ & 0.10 & $2.12 * *$ & $-0.15^{*}$ & $9.33 * *$ & $13.06 * *$ & $0.55^{*}$ & 0.36 & $8.84 * *$ & $7.43 * *$ & 1.00 \\
\hline 2 & $\mathrm{JMSA}_{5} 20175$ & $-0.32 *$ & $0.25 *$ & $-0.15^{*}$ & $0.90 *$ & 0.90 & $-4.52 *$ & $11.57 * *$ & -0.42 & -0.39 & -3.12 & $-4.33 * *$ & -1.16 \\
\hline 3 & $\mathrm{JMSA}_{1} 20181$ & $-1.18^{* *}$ & $-0.83^{* *}$ & 0.14 & $-1.07 *$ & $-1.07 *$ & $-9.83 * *$ & $-22.50 * *$ & $-2.34 * *$ & $-0.71 * *$ & $-13.02 * *$ & -7.40 & 0.21 \\
\hline \multirow[t]{2}{*}{4} & ICMA $_{1} 15666$ & $0.93 * *$ & $-0.55^{* *}$ & $0.19 * *$ & -0.16 & -0.16 & $5.02 *$ & -2.13 & $2.21 * *$ & $0.73 * *$ & $7.30 * *$ & 4.30 & 0.37 \\
\hline & S.E. \pm & 0.13 & 0.11 & 0.05 & 0.32 & 0.05 & 1.75 & 1.74 & 0.22 & 0.19 & 1.92 & 1.07 & 0.74 \\
\hline \multicolumn{14}{|c|}{ Males } \\
\hline 1 & $\mathrm{~J}-2584$ & $-0.72 *$ & $-0.49 *$ & -0.08 & 0.13 & -0.18 & -5.40 & -1.81 & $0.91 *$ & 0.42 & $-9.98 *$ & $-9.17 * *$ & -2.18 \\
\hline 2 & $\mathrm{~J}-2591$ & $3.03 * *$ & 0.29 & -0.02 & 0.29 & $-0.22 *$ & $-8.29 *$ & $12.41 * *$ & $3.99 * *$ & $-1.01 * *$ & -4.82 & $-11.61 * *$ & $-5.18 * *$ \\
\hline 3 & J- 2598 & $2.63 * *$ & $0.92 * *$ & 0.15 & $2.00 * *$ & 0.12 & $10.18 *$ & $20.34 * *$ & $2.82 * *$ & 0.40 & $9.87 *$ & $8.55 * *$ & 1.36 \\
\hline 4 & $\mathrm{~J}-2605$ & $1.19 * *$ & 0.02 & -0.01 & 0.29 & 0.19 & 2.91 & $8.77 *$ & 0.74 & 0.18 & 3.28 & $5.17 *$ & 2.05 \\
\hline 5 & 52-SB-18 & $-3.31 * *$ & 0.32 & -0.01 & $-1.55^{*}$ & $0.29 *$ & -3.84 & $-11.63 * *$ & $-4.34 * *$ & -0.11 & -3.08 & 3.19 & $3.55^{*}$ \\
\hline 6 & 72-SB-18 & $-1.39 * *$ & -0.26 & -0.08 & 1.77 & 0.01 & 3.43 & $-8.33^{*}$ & $-1.76 * *$ & 0.40 & 0.92 & $4.35^{*}$ & 2.27 \\
\hline 7 & 88-SB-18 & $0.97 * *$ & -0.09 & -0.12 & $-2.54 * *$ & $-0.27 *$ & $-12.26^{* *}$ & $-8.44 *$ & -0.68 & -0.65 & $8.50 * *$ & $-9.56 * *$ & -2.53 \\
\hline 8 & 92- SB- 18 & -0.22 & $0.67 * *$ & 0.17 & -0.45 & $0.21 *$ & 6.11 & $9.26 * *$ & $-1.01 *$ & 0.40 & $7.67 * *$ & $5.06^{*}$ & 0.61 \\
\hline \multirow[t]{2}{*}{9} & 120-SB-18 & 0.03 & $-0.76^{* *}$ & -0.01 & 0.05 & -0.15 & $7.16^{*}$ & $-20.57 * *$ & $0.68^{*}$ & 0.03 & $6.48 *$ & 4.02 & 0.07 \\
\hline & S.E. \pm & 0.20 & 0.16 & 0.08 & 0.48 & 0.08 & 2.63 & 2.61 & 0.33 & 0.28 & 2.89 & 1.61 & 1.11 \\
\hline
\end{tabular}

$*, * *$ Significant at $5 \%$ and $1 \%$ levels, respectively

Table.4 Top three crosses recorded sca effects for different characters

\begin{tabular}{|c|c|c|c|c|c|c|c|}
\hline Characters & Crosses & & Characters & Characters & Crosses & Characters & Crosses \\
\hline $\begin{array}{l}\text { Days to } \\
50 \% \\
\text { flowering }\end{array}$ & $\begin{array}{l}\text { ICMA }_{1}-15666 \times 88-S B-18 \\
\text { JMSA }_{4}-20172 \times 52-S B-18 \\
\text { JMSA }_{1}-20181 \times \text { J J-2591 }\end{array}$ & $\begin{array}{c}\text { Ear head length } \\
\text { (cm) }\end{array}$ & - & $\begin{array}{l}\text { Plant } \\
\text { height } \\
(\mathbf{c m})\end{array}$ & $\begin{array}{l}\text { JMSA5-20175 x 120-SB-18 } \\
\text { ICMA1-15666 x 72-SB-18 } \\
\text { JMSA5-20175 x J-2584 }\end{array}$ & $\begin{array}{l}\text { Dry fodder } \\
\text { yield per } \\
\text { plant (g) }\end{array}$ & $\mathrm{JMSA}_{1}-2181 \times \mathrm{J}-2605$ \\
\hline $\begin{array}{l}\text { Number of } \\
\text { nodes on } \\
\text { main stem }\end{array}$ & $\begin{array}{l}\text { JMSA5-20175 x 120-SB-18 } \\
\text { JMSA5-20175 x 92-SB-18 }\end{array}$ & $\begin{array}{l}\text { Ear head girth } \\
(\mathrm{cm})\end{array}$ & $\begin{array}{l}\text { JMSA1-20181 x J-2584 } \\
\text { JMSA1-20181 x 120-SB-18 } \\
\text { ICMA1-15666 x 88-SB-18 }\end{array}$ & $\begin{array}{l}\text { Days to } \\
\text { maturity }\end{array}$ & $\begin{array}{l}\text { JMSA4-20172 x J-2584 } \\
\text { JMSA1-20181 x J-2591 } \\
\text { JMSA1-20181 x J-2598 }\end{array}$ & $\begin{array}{l}\text { Grain yield } \\
\text { per plant }(\mathrm{g})\end{array}$ & $\begin{array}{l}\text { JMSA4-20172 × J-2591 } \\
\text { JMSA1-20181 x J-2584 } \\
\text { JMSA5-20175 x J-2598 }\end{array}$ \\
\hline $\begin{array}{l}\text { Number of } \\
\text { effective } \\
\text { tillers/ } \\
\text { plant }\end{array}$ & $\begin{array}{l}\text { JMSA5-20175 x J-2598 } \\
\text { ICMA1-15666 x 92-SB-18 } \\
\text { ICMA1-15666 x J-2605 }\end{array}$ & $\begin{array}{c}\text { Ear head weight } \\
\text { (g) }\end{array}$ & $\begin{array}{l}\text { JMSA1-20181 x J-2584 } \\
\text { JMSA5-20175 x 88-SB-18 } \\
\text { JMSA4-20172 x J-2591 }\end{array}$ & $\begin{array}{l}\text { 1000- } \\
\text { grain } \\
\text { weight } \\
\text { (g) }\end{array}$ & $\begin{array}{l}\text { JMSA5-20175 x J-2598 } \\
\text { JMSA4-20172 x J-2591 } \\
\text { ICMA1-15666 x J-2605 }\end{array}$ & $\begin{array}{c}\text { Harvest } \\
\text { index }(\%)\end{array}$ & $\mathrm{JMSA}_{1}-20181 \times \mathrm{J}-2584$ \\
\hline
\end{tabular}


Table.5 The best five specific cross combination with their resulting sca effects for grain yield and other component characters including gca effects of their parents for grain yield per plant

\begin{tabular}{|c|c|c|c|c|c|c|}
\hline \multirow[t]{2}{*}{ Crosses } & \multirow[t]{2}{*}{$\begin{array}{l}\text { Grain yield per } \\
\text { plant (g) }\end{array}$} & \multirow{2}{*}{$\begin{array}{c}\text { sca effect for } \\
\text { grain yield per } \\
\text { plant }\end{array}$} & \multicolumn{2}{|c|}{$\begin{array}{c}\text { gca effect for grain yield per } \\
\text { plant }\end{array}$} & \multirow{2}{*}{$\begin{array}{c}\text { Heterosis (\%) over } \\
\text { Standard check (GHB- } \\
732)\end{array}$} & \multirow{2}{*}{$\begin{array}{l}\text { Significant desirable } \\
\text { sca effects for } \\
\text { component traits }\end{array}$} \\
\hline & & & Female & Male & & \\
\hline JMSA5-20175 x J-2598 & 74.17 & $11.03 *$ & $-4.33 * *$ & $8.55^{* *}$ & $27.43 * *$ & 3,9 \\
\hline $\begin{array}{l}\text { ICMA } 1 \text {-15666 x 92-SB- } \\
18\end{array}$ & 73.93 & 5.65 & $4.30 *$ & $5.06^{*}$ & $27.03 * *$ & 3 \\
\hline ICMA $_{1}-15666$ x J-2605 & 72.67 & 4.27 & $4.30 *$ & $5.17 *$ & $24.86 * *$ & $3,8,9$ \\
\hline $\mathrm{JMSA}_{4}-20172$ x J-2591 & 72.20 & $17.45 * *$ & $7.43 * *$ & $-11.61 * *$ & $24.05 * *$ & $3,6,9$ \\
\hline $\begin{array}{l}\mathrm{JMSA}_{4}-20172 \text { x 52-SB- } \\
18\end{array}$ & 72.00 & 2.45 & $7.43 * *$ & 3.19 & $23.71 * *$ & - \\
\hline
\end{tabular}

Where, *** significant at $5 \%$ and $1 \%$ levels, respectively

Where,

\begin{tabular}{|c|c|c|c|c|c|c|c|c|c|c|c|}
\hline 1 & $=$ & $\begin{array}{c}\text { Days to } 50 \% \\
\text { flowering }\end{array}$ & 2 & $=$ & $\begin{array}{c}\text { Number of nodes on main } \\
\text { stem }\end{array}$ & 3 & $=$ & $\begin{array}{c}\text { Number of effective tillers/ } \\
\text { plant }\end{array}$ & 4 & $=$ & Ear head length $(\mathrm{cm})$ \\
\hline 5 & $=$ & Ear head girth $(\mathrm{cm})$ & 6 & $=$ & Ear head weight (g) & 7 & $=$ & Plant height $(\mathrm{cm})$ & 8 & $=$ & Days to maturity \\
\hline 9 & $=$ & $\begin{array}{l}\text { 1000-grain weight } \\
\text { (g) }\end{array}$ & 10 & $=$ & Dry fodder yield/plant (g) & 11 & $=$ & Harvest index (\%) & & & \\
\hline
\end{tabular}


These results are supported by Acharya (2017), Katba (2017), Solanki et al., (2017), Kanfany et al., (2018) and Kumawat et al., (2019).

From the present inheritance studied traits, we can conclude that both additive and nonadditive gene actions recorded equal importance as half of the traits governed by non-additive gene action and other half by additive gene action. From ongoing discussion, it was suggested that best two promising crosses namely $\mathrm{JMSA}_{5}-20175$ x J2598 and $\mathrm{JMSA}_{4}-20172 \times \mathrm{J}-2591$ exhibited high per se performance, high magnitude of heterobeltiosis as well as standard heterosis, significant and positive sca effects in desired direction for grain yield per plant and some other component traits. Therefore, these two crosses could be further evaluated over years and locations to exploit for commercial cultivation or utilized in future breeding programme to obtain desirable transgressive segregants for the development of superior genotypes or inbreeds.

\section{References}

Acharya, Z. R. 2017. Study on heterosis and combining ability analysis using male sterile lines in pearl millet [Pennisetum glaucum (L.) R. Br.]. Unpublished M.Sc. (Agri.) thesis submitted to JAU, Junagadh.

Badurkar, S. B., Pole, S. P., Toprope, V. N. and Ingle, N. P. 2018. Combining ability for grain yield and its related traits in pearl millet [Pennisetum glaucum (L.) R. Br.]. Int. J. Curr. Microbiol. App. Sci., 6: 956-961.

Gavali, R. K., Kute, N. S., Pawar, V. Y. and Patil, H. T. 2018. Combining ability analysis and gene action studies in pearl millet [Pennisetum glaucum (L.) R. Br.]. Elect. J. P. Breed., 9(3): 908-915.

Jethva, A. S., Raval, L., Madariya, R. B.,
Mehta, D. R. and Mandavia, C. 2012. Heterosis for grain yield and its related characters in pearl millet. Elect. J. Pl. Breed., 3(3): 848-852.

Kanfany, G., Fofana, A., Tongoona, P., Danquah, A., Offici, S., Danquah, E. and Cisse, N. 2018. Estimates of combining ability and heterosis for yield and its related traits in pearl millet inbred lines under downy mildew prevalent areas of Senegal. Int. J. Agro., Article ID-34390390.pp-12.

Katba, P. J. 2017. Studies on line $\mathrm{x}$ tester analysis over environments in pearl millet [Pennisetum glaucum (L.) R. Br.]. Unpublished Ph.D. (Agri.) thesis submitted to AAU, Anand.

Krishnan, M. R., Bhadauria, H. S., Patel, M. S., Patel, Y. N. and Gami, R. A. 2017. Genetic analysis in pearl millet [Pennisetum glaucum (L.) R. Br.]. Int. J. Curr. Microbiol. App. Sci. 6(11): 900-907.

Kumawat, K. R., Gupta, P. C. and Sharma, N. K. 2019. Combining ability and gene action studies in pearl millet using line $\mathrm{x}$ tester analysis under arid conditions. Int. J. Curr. Microbiol. App. Sci., 8(4): 976-984.

Ladumor, V. L., Mungra, K. D., Parmar, S. K., Sorathiya, J. S. and Vansjaliya, H. G. 2018b. Grain iron, zinc and yield genetics in pearl millet [Pennisetum glaucum (L.) R. Br.]. Int. J. Curr. Microbiol. App. Sci., 7(9): 242-250.

Mungra, K. S. 2014.Heterosis and combining ability analysis for grain yield and its components in pearl millet [Pennisetum glaucum (L.) R. Br.]. Unpublished M.Sc. (Agri.) thesis submitted to JAU, Junagadh.

Nandaniya, K. U., Mungra, K. D. and Sorathiya, J. S. 2016. Assessment of combining ability for yield and micro nutrient in pearl millet [Pennisetum glaucum (L.) R. Br.]. Elect. J. pl. 
Breed., 7(4): 1084-1088.

Patel, S. M. 2012. Genetic analysis of grain yield and its component characters in pearl millet [Pennisetum glaucum (L.) R. Br.]. Unpublished M. Sc. (Agri.) thesis submitted to Anand Agricultural University, Anand.

Sehgal, A., Telang, S., Passah, S. and Jyothi, M. 2004. Maternal and neonatal profile and immediate outcome in extremely low birth weight babies in Delhi. Trop. Doctor, 34(3): 165-168.

Singh, F., Nainawatee, H., Khairwal, I., Rai,
K., Andrews, D. and Harinarayana, G. 1999. Grain quality traits, in pearl millet breeding, Eds Khairwal I. S., Rai K. N., Andrews D. J., Harinarayana G. editors. (New Delhi: Vijay Primlani).

Solanki, K., Bhinda, M; Gupta, P., Saini, H. and Saini, L. 2017. Combining ability and gene action studies for grain yield and component characters in pearl millet [Pennisetum glaucum (L.) R. Br.] under arid condition of Rajasthan. Int. $J$. Pure App. Biosci. 5(4): 2121-2129.

\section{How to cite this article:}

Patel, K. N., K. K. Dhedhi, H. Ramani and Vaghela, U. U. 2021. Genetic Analysis in Pearl millet Using Line x Tester Design. Int.J.Curr.Microbiol.App.Sci. 10(01): 1202-1209.

doi: https://doi.org/10.20546/ijcmas.2021.1001.144 